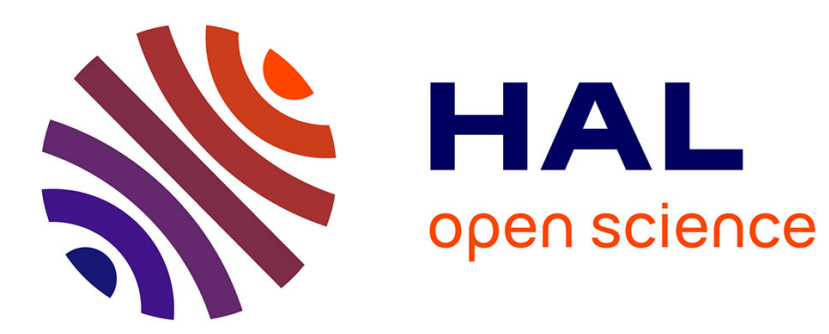

\title{
La combinaison des outils de communication à l'interface de la relation-client dans les TPE
}

Jérôme Denis

\section{To cite this version:}

Jérôme Denis. La combinaison des outils de communication à l'interface de la relation-client dans les TPE. Réseaux : communication, technologie, société, 2003, 121, pp.71-92. halshs-00265643

\section{HAL Id: halshs-00265643 \\ https://shs.hal.science/halshs-00265643}

Submitted on 19 Mar 2008

HAL is a multi-disciplinary open access archive for the deposit and dissemination of scientific research documents, whether they are published or not. The documents may come from teaching and research institutions in France or abroad, or from public or private research centers.
L'archive ouverte pluridisciplinaire HAL, est destinée au dépôt et à la diffusion de documents scientifiques de niveau recherche, publiés ou non, émanant des établissements d'enseignement et de recherche français ou étrangers, des laboratoires publics ou privés. 


\title{
La combinaison des outils de communication à l'interface de la relation-client dans les TPE
}

\author{
Jérôme DENIS \\ TELECOM ParisTech \\ Département Sciences Économiques et Sociales \\ 46, rue Barrault \\ F-75634 Paris Cedex 13 \\ 0145817611 \\ http://ses.enst.fr/denis
}

\section{Résumé}

À partir d'une enquête sur les modalités de la relation à la clientèle dans les petites entreprises, cet article souligne le caractère combinatoire des usages des outils de communication. Les personnes en charge de répondre aux clients, de les démarcher, ou de produire et de faire circuler des informations les concernant s'appuient en effet sur des chaînes techniques qui articulent des dispositifs hétérogènes. Interroger les valeurs pragmatiques qui sont attachées à ces différents outils permet de montrer en quoi de telles chaînes participent à élaborer une relation-client pluridimensionnelle, qui ne se réduit pas aux seuls échanges interpersonnels.

Denis, J. 2003. La combinaison des TIC à l'interface de la relation-client dans les TPE, Réseaux (121), p. 71-92. 


\section{introduction : TIC, relation-client et petites entreprises}

Au premier abord, les petites entreprises nous invitent à insister, du fait même de leur taille, sur l'importance des liens personnels, de la proximité et de la confiance dans les relations qu'elles entretiennent avec leurs clients ${ }^{1}$. Le petit nombre de salariés, le caractère localisé de leurs activités, leur contact permanent avec le "terrain " les désigneraient comme un cas paradigmatique pour souligner l'« encastrement social » de l'activité économique ${ }^{2}$. Pourtant, dire que la relation-client repose uniquement dans les petites entreprises sur des échanges personnels, où l'interconnaissance des protagonistes ne tient que sur une histoire commune et une confiance lentement élaborée, reviendrait à passer sous silence une grande partie du travail relationnel qui y est mis en œuvre. Pour être appréhendé dans son épaisseur, celui-ci doit pouvoir être sorti du modèle unique de la relation en face-à-face et être questionné au regard de la place qu'y tiennent les nombreux équipements qui l'accompagnent. Le téléphone, les cahiers d'appels, les logiciels de gestion, le courrier électronique, le fax, etc. sont des outils courants dans les petites entreprises. Ils équipent les échanges et participent à les mettre en forme. Ils méritent une attention toute particulière, notamment parce qu'ils apparaissent comme un point aveugle de l'étude des relations que les petites entreprises entretiennent avec leurs clients, qui tendent à se focaliser sur les rencontres en coprésence et les liens qu'elles alimentent. Quelle est la place de ces dispositifs dans ces relations ? Comment participent-ils à ces liens "chauds ", durables, à ces échanges informels, souvent décrits comme ciment de la pérennité de ce type d'entreprises et incompatibles avec les formes "froides ", standardisées, des marchés à grande échelle ? Dans cet article, j'essaierai de montrer que, s'ils prennent des formes différentes des dispositifs complexes que les grandes entreprises dédient à la relation-client ${ }^{3}$, de tels équipements ont aussi leur importance dans les petites entreprises. Cette perspective m'amènera à relativiser la figure unique de la relation informelle avec le client. Nous verrons qu'à celle-ci s'ajoutent d'autres formes de liens, d'autres registres relationnels, que les technologies de l'information et de la communication participent à articuler.

Pour répondre à cette ambition, l'enquête qui a été mise en place s'est attaché à produire des monographies, effectuées à partir de deux ou trois entretiens par entreprise. Ces entretiens ont été menés auprès de différentes personnes: une secrétaire ou une standardiste, un employé (le plus souvent en contact avec les clients), et le gérant. Ils ont porté sur l'équipement général de l'entreprise ; sur les types de relation qu'elle entretient avec ses clients, ses fournisseurs et ses partenaires; et sur les usages concrets des différents outils disponibles. La population étudiée est composée de huit entreprises de Haute-Garonne comprenant entre trois et dix-neuf salariés (cf. encadré). Le choix de ces entreprises s'est effectué à partir de la base commerciale d'une entreprise de télécommunication. Toutes avaient fait l'acquisition du logiciel que nous appellerons ici CustomScreen, qui permet un couplage téléphonie/informatique afin de faire apparaître automatiquement lors de l'appel d'un client sa fiche détaillée sur l'écran de l'ordinateur. Le but premier de l'enquête était de comprendre la place que prenait cet outil dans les

1. Cet article rend compte d'une enquête commanditée par France Télécom R\&D, menée avec l'aide de certains étudiants de l'IUP de sociologie appliquée de l'université Toulouse II. Je tiens à remercier tout particulièrement Manon Meunier et Stéphanie Perret pour leur investissement dans le travail empirique et dans les premiers temps de l'analyse, ainsi que Franck Cochoy et Alexandre Mallard qui ont encadré une partie de ce travail. Je remercie enfin Sandrine Barrey, Philippe Cotnoir, David Martin, David Pontille et Aurélie Lachèze pour leurs remarques constructives lors de la lecture de versions intermédiaires de ce papier.

2. GRANOVETTER, 1985

3. MALLARD, 2002 
usages communicationnels de ces entreprises. Mais ce but n'a pu être atteint : si toutes les entreprises interrogées étaient en possession de l'outil, seules deux d'entre elles l'utilisaient (à des degrés très différents). Cette absence d'usage qui aurait pu constituer un « raté » empirique a en fait permis de mettre l'accent sur une dimension primordiale de notre questionnement. La non-intégration de CustomScreen soulève en effet la question de la consistance des technologies déjà en place et des éventuelles formes de combinaison et d'interdépendance qui se créent entre elles. Dans les entreprises étudiées, la prise de contact d'un client, les demandes de renseignements, la prospection, ou encore la diffusion des informations auprès des collègues, sont autant d'activités communicationnelles qui sont enchevêtrées par l'intermédiaire d'une combinaison de dispositifs hétérogènes, additionnés en quelque sorte les uns aux autres. Au-delà de la question de l'appropriation d'un dispositif technique spécifique, et des bricolages qu'elle ne manque pas d'occasionner, l'absence d'intégration de CustomScreen dans les pratiques invite à interroger ces nombreuses combinaisons qui lient les outils entre eux dans l'activité. J'insisterai donc ici sur ces chaînes techniques qui équipent la relationclient dans les petites entreprises. Cette perspective m'amènera à reconstituer le détail des chaînages quotidiens des technologies de l'information et de la communication et me permettra de questionner les valeurs pragmatiques qui sont attachées aux différents dispositifs ${ }^{4}$.

\section{Population}

L'enquête a été menée auprès de huit entreprises de Haute-Garonne. Une agence immobilière ( 3 personnes) ; une société de services en ingénierie informatique, agence d'une entreprise dont le siège est à Strasbourg (19 personnes); entreprise de fret (10 personnes); une association spécialisée dans la formation (8 personnes); une coopérative de conseil juridique (6 personnes); une entreprise de vente de logiciels médicaux qui fait aussi de l'assistance téléphonique (6 personnes), une entreprise de fournitures de bureau (5 personnes) ; et une entreprise de plomberie spécialisée dans la réparation et l'installation de structure de plomberie, de chauffage et de climatisation (11 personnes).

\section{Des chaînes techniques pour la relation-client}

À propos d'une étude empirique en milieu hospitalier, B. Fraenkel souligne la grande diversité des écrits qui sont produits et qui circulent au quotidien dans le travail ${ }^{5}$. En détaillant très finement le contenu et la forme des documents qui composent le " cahier de transmission ", l'article montre que les types mêmes d'écritures y sont très variés (dactylographiée, manuscrite, signes, abréviations, signatures...), ainsi que les énonciateurs qui y participent. Pour rendre compte de l'utilisation qui est faite de ces écrits, leur circulation, leur copie, les retours dont ils sont l'objet, B. Fraenkel élabore la notion de "chaînes d'écriture », dont je propose de m'inspirer pour mieux comprendre les combinaisons d'outils communicationnels dans les petites entreprises.

On peut ainsi mettre en évidence des "chaînes d'écriture " qui assurent à tout ou partie d'un texte une diffusion sur divers supports et surtout qui permettent à un même contenu informatif de remplir des rôles différents. (p. 246)

Le premier intérêt de cette notion est de mettre en lumière l'articulation des supports qui est en jeu dans la circulation des écrits hospitaliers. Ce point souligne d'abord que les

4. C'est-à-dire les capacités d'action qui leur sont reconnues.

5 . FRAENKEL, 2001 
chaînes d'écritures ne sont pas de simples successions hasardeuses de documents qui n'auraient pas de contact les uns avec les autres. Ces derniers sont mobilisés en fonction les uns des autres (ils s'en-chaînent) et leurs utilisations dessinent un fil d'action collective. Mais au-delà de cette vision d'une activité de communication par étapes successives liées les unes aux autres, c'est la question même des propriétés matérielles des supports de la communication et de leur force pragmatique que $\mathrm{B}$. Fraenkel soulève.

Cette dimension est aussi centrale dans les nombreuses chaînes techniques qui articulent les outils de communication dans les petites entreprises. Pour mieux en saisir les enjeux, je propose de partir d'un exemple précis : celui de l'entreprise de fret. L'activité de cette entreprise se concentre sur la mise en relation de personnes souhaitant faire transporter des marchandises et de transporteurs proposant des camions et des trajets en Europe. L'entreprise a donc affaire à deux types de clients, offreurs et demandeurs. Le traitement de cette double relation passe par une série d'outils pour la communication et la circulation d'informations dont le maniement ponctue l'activité quotidienne.

C'est par téléphone, dans la très grande majorité des cas, que les clients demandeurs entrent en contact avec l'entreprise. Mais ce premier échange est déjà doublement équipé. Au téléphone est associé le logiciel CustomScreen qui permet une présentation du numéro améliorée : Iors d'un appel d'un client connu, une fiche le concernant apparaît sur l'écran de l'ordinateur, qui affiche ses principales caractéristiques (nom, adresse, numéro de téléphone, mais aussi type de marchandises, derniers trajets, prix habituels, etc.). Par ailleurs, le logiciel est aussi sollicité pour produire régulièrement une trace des différents appels qui permet par exemple de compter le nombre d'appels déjà passés auprès de tel ou tel transporteur, pour ne pas le submerger dans un temps trop court. La combinaison des outils de communication se poursuit, sur le versant informatique. Pour répondre à la demande du client (telle quantité et tel type de marchandises doit être livrée à telle date, à tel endroit), les employés se tournent vers une base de données élaborée sur un tableur sur laquelle est répertoriée une série d'informations concernant les transporteurs. Par une fonction de tri des caractéristiques pertinentes, apparaît sur l'écran le (ou les) transporteur(s) susceptible(s) de faire l'affaire pour l'occasion. Lorsque celui-ci est sélectionné, les deux parties sont contactées par téléphone pour confirmer la mise en relation. À la suite de cette transaction, un " bon pour accord " est envoyé à chacun par fax, retournés immédiatement une fois signé. Cet échange de fax peut être suivi d'un dernier appel téléphonique qui règle les éventuels détails de coordination.

Du point de vue de l'entreprise, il faut ajouter d'autres outils à cette combinaison. Tout d'abord, des dossiers "papier » sont constitués pour chaque client et pour chaque transaction. Ces dossiers sont composés d'informations directement extraites de la base de données via leur impression, des fax signés et des éventuels journaux d'appels produits par CustomScreen. À ces archives sur papier s'ajoute enfin un système de sauvegarde informatique quotidien qui stocke sur des disques durs dédiés les informations de la base de données.

Cet exemple montre à quel point les usages des technologies de l'information et de la communication ne sont pas isolés les uns des autres. Dans l'entreprise de fret, on ne fait pas qu'utiliser une variété d'outils : on passe de l'un à l'autre, et ces passages (résumés dans la figure 1) ont un sens pour l'activité. Ils participent pleinement au déroulement de la relation avec les clients ${ }^{6}$.

\footnotetext{
6. Bien entendu, le cas de cette entreprise présente des spécificités non négligeables. Le travail d'alignement entre clients offreurs et demandeurs, le rythme soutenu des échanges pour conclure un accord au plus vite, sont autant de dimensions qui cadrent la combinaison des outils. Mais ces spécificités ne doivent pas être considérées comme les conditions sine qua non de la mise en œuvre de chaînes techniques. Nous le verrons au fil des extraits d'entretien qui nourrissent cet
} 




Figure 1. La combinaison des outils dans l'entreprise de fret

On voit à travers cet exemple que la question des supports communicationnels est fondamentale pour comprendre ce qui est en jeu dans le chaînage des technologies de communication. Le passage du téléphone au fax ou au logiciel de base de données, l'affichage de fiches dur l'écran, la saisie d'informations sur un cahier d'appels, le rangement de documents dans un classeur d'archives font tour à tour basculer la relation et les différentes dimensions qui en sont retenues sur des supports différents. Ces supports opèrent une mise en forme particulière des informations, et les aménagent en appuis possibles pour l'action. Les couples informations-supports constituent ainsi des " artefacts cognitifs ${ }^{7}$ au sens large qui permettent d'externaliser la mémoire, de partager certaines dimensions d'un échange interpersonnel, etc.

Un autre apport de la notion de chaînes d'écriture est d'offrir la possibilité de séparer le contenu informatif et les "fonctions" qui peuvent lui être attribuées en situation. Les passages d'un support à un autre ne sont pas seulement des moyens de faire circuler une information, ou de la rendre disponible dans telles situations à telles personnes. Comme le montrent M. Grosjean et M. Lacoste, les supports eux-mêmes ont des «propriétés représentationnelles ${ }^{8}$. Le basculement entre deux supports transforme au sens propre les énoncés et leur(s) signification(s). C'est ce point qu'il est important d'affiner dans le cas de la relation-client telle qu'elle est équipée et organisée dans les petites entreprises. Quelles sont les différentes significations qui ont cours selon les dispositifs de communication qui participent aux chaînages ? Quelle « direction " prend la relation avec les clients dans chacun des maillons de la chaîne?

Pour répondre à ces questions, et saisir l'enjeu pragmatique des combinaisons techniques, il est nécessaire d'élargir la perspective des " chaînes d'écriture » pour saisir dans un même mouvement les supports de communication orale (téléphone, guichet...) et écrite (cahier d'appels, documents électroniques, fiches, petits papiers éphémères, fax...). Comme le laissait entendre l'exemple développé plus haut, les technologies de l'information et de la communication seront appréhendées ici d'un point de vue très large, qui rassemble aussi bien les formats hétérogènes de communication, que les générations de dispositifs. Enfin, plutôt que de questionner les "fonctions" communicationnelles telles qu'elles sont modifiées à travers les chaînages, je propose de me concentrer sur les registres de l'activité dans lesquels sont engagés à la fois les personnes et les énoncés qu'elles manipulent. Ce léger déplacement de la focale nous permettra de comprendre comment les chaînes techniques participent à l'épaisseur interactionnelle de la relation avec les clients.

article, si leur forme et le nombre d'outils qu'ils concernent peuvent varier, le principe même des chaînages est très général.

7. NORMAN, 1993

8. GROSJEAN \& LACOSTE, 1998 


\section{Chainages et registres de l'activité : l'articulation des temporalités}

Questionner les chaînes des technologies de communication est un moyen d'appréhender la diversité des dispositifs relationnels qui équipent les rencontres entre les entreprises et leurs clients sans définir a priori leur position et leur force. Ce point de vue invite à interroger les valeurs qui sont attachées dans l'usage aux technologies de communication. Ces valeurs se traduisent par l'importance que prennent certains dispositifs dans des registres d'activité spécifiques. L'attention portée à l'enchaînement des outils en situation met ici en lumière le caractère composite de la relation-client dans les petites entreprises. Les multiples passages d'un support à un autre opèrent une combinaison de registres parfois antagonistes. Dans le cas de la relation-client, ces registres peuvent être caractérisés par des types de temporalités spécifiques.

\section{Rapidité et réactivité}

La rapidité de réaction de l'entreprise face à la demande est une dimension centrale de la relation-client telle qu'elle est décrite par les personnes interrogées. Que ce soit pour l'association, l'entreprise de fourniture de bureau, l'agence immobilière, ou encore le plombier, s'il est un impératif, c'est bien celui de répondre " vite et bien » aux clients. De ce point de vue, le téléphone est l'équipement privilégié : il est considéré par beaucoup comme l'unique outil qui permet une communication simple et efficace, élément primordial pour rester concurrentiel sur le marché.

Le téléphone, c'est un outil qui me permet essentiellement de réagir vite. Donc j'ai une information, je joins rapidement la personne. Ça me donne un temps de réaction rapide, et aujourd'hui, les affaires il faut qu'elles se fassent rapidement parce que vu la concurrence que nous avons, vu le peu de produits que malgré tout nous avons qui gravitent, je ne peux me permettre de rater une affaire qui est là par manque de rapidité. [Et dans le détail c'est quoi la rapidité ?] La rapidité, un exemple, c'est que j'ai une information de terrain, je l'ai au moment $X$, au moment $X$ plus une minute, je contacte un client pour ce produit-là. (Commercial, agence immobilière)

Parce ce qu'il permet de joindre la personne désirée sur un rythme dialogique, contrairement au courrier électronique ou au fax qui n'impliquent pas de réponse immédiate, le téléphone est considéré des deux côtés de la communication (entreprises et clients) comme un moyen sûr de régler une affaire ou un litige dans les meilleures conditions ${ }^{9}$.

Lorsque cette temporalité courte est la seule impliquée dans la relation, les chaînes techniques sont donc quasiment inexistantes. Un seul outil vient jouer un rôle central, quasi-hégémonique. Mais lorsque l'interaction avec le client ne nécessite pas seulement une réaction directe dans la conversation, d'autres dispositifs peuvent rentrer en jeu. Le fax est ainsi l'un des outils le plus souvent couplé au téléphone.

On utilise très souvent le fax en fait. À la fois pour recevoir et pour émettre des documents. Surtout des devis. Beaucoup de gens, les syndics ou aussi les particuliers veulent qu'on leur faxe les devis à leur domicile ou à leur bureau. (Secrétaire, entreprise de plomberie)

On trouve aussi des chaînes plus sophistiquées, comme dans l'entreprise de vente de fournitures de bureau où la standardiste réceptionne les commandes par téléphone et les inscrit dans un logiciel de gestion qui relie en réseau l'entreprise à son unique fournisseur. Cette procédure permet de livrer le client sous vingt-quatre heures si la commande a été passée avant midi.

${ }^{9}$. Ces éléments ont été bien montré par C. Licoppe (2001) dans le cas de la vente à distance. 
C'est un système qu'a installé notre fournisseur. Le logiciel de gestion prépare les commandes [enregistrées manuellement après un coup de téléphone ou un e-mail] et les met dans une sorte de boîte aux lettres. Les commandes restent là, et toutes les trois heures, le fournisseur se branche chez nous, ouvre la boîte aux lettres, prend ce qu'il y a dedans, repart avec et l'exploite. (Directeur, entreprise de fournitures de bureau)

Pourtant, les choses sont rarement si simples. Dans la plupart des cas, ce rythme d'interaction question-réponse-clôture ne suffit pas. À moins de n'avoir à faire qu'à des clients occasionnels que l'on est sûr de ne jamais revoir, les relations sont généralement prises dans des temporalités plus riches. C'est ici que les premières chaînes complexes apparaissent, qui font jouer temps court de l'interaction directe et temps plus long de la trace et de la mémoire.

\section{La trace au service de la réactivité}

La rapidité et la réactivité de l'entreprise face à ses clients ne s'épuisent pas dans l'ici et maintenant de la situation de communication. Certaines TPE, à l'image des grandes entreprises, mettent ainsi en place des systèmes qui permettent de garder une trace des interactions passées, ou de certaines caractéristiques du client lui-même, qui peuvent être réactivées en situation pour accentuer encore la rapidité de réaction.

Par exemple, L. va m'appeler et il va me dire combien je dois, parce qu'il vient ce soir pour faire l'état des lieux, il va me dire " combien je dois ». Eh bien [en regardant son écran], je lui dis: vous m'amenez un chèque de 250 euros, voilà. "Ça correspond à quoi monsieur ? ", ça correspond au loyer de Mars au prorata de 250, les provisions sur charge pour 277 , les garanties qui courent pour 270 euro, etc. La dame qui est venue tout à l'heure qui m'a porté son chèque qui n'était pas rempli, elle l'avait signé, je lui ai regardé son compte et puis j'ai tout de suite vu combien je devais lui rembourser. Donc il y a cette immédiateté... (Gérant, agence immobilière)

Qu'il s'agisse d'une demande de fret, d'une commande, d'une demande d'information ou d'un dépannage informatique, le téléphone est donc enchaîné à d'autres moyens d'information sur ou pour le client qui permettent de rendre accessible au moment même de l'interaction son identité, ce qu'il veut, ce qu'on lui a déjà proposé, etc. Et comme le suggère cet exemple, l'obligation de rapidité favorise tout particulièrement l'usage des systèmes d'information informatisés, et plus rarement celui de traces papiers.

Mais, à y regarder de plus près, cet enchaînement repose en fait sur deux types d'opérations, deux chaînages. Celui que nous venons de citer consiste à agencer le téléphone (ou le face-à-face au guichet) et des outils pour la plupart informatisés qui affichent sur un écran certaines caractéristiques du client. La chaîne relie donc un outil de communication à un artefact cognitif qui stocke et matérialise une mémoire externe de la relation, susceptible d'être réactivée lors d'une autre situation de communication. Or, pour que cette chaîne puisse fonctionner, il faut qu'elle repose sur une autre, répétée de nombreuses fois : celle qui permet d'intégrer les informations « dans » l'artefact. Pour que la mémoire externe soit pertinente, il faut qu'on y dépose des souvenirs... L'autre chaînage consiste donc à inscrire dans les outils prévus à cet effet les éléments de caractérisation des clients pendant, ou à la suite de son appel.

Quand une personne m'appelle, quand elle est intéressée par une formation quel que soit le domaine, qu'elle me pose des questions... je demande son adresse. On a un cahier qui est ici, donc à chaque fois je prends les adresses des gens qui téléphonent et quand j'ai un petit moment, je rentre dedans [dans un logiciel de base de données]. Je rentre dans la base, et comme ça cette année, je prévois de leur envoyer le programme 2002-2003. (Secrétaire, association) 
Nous le voyons, en passant du téléphone au logiciel, puis du logiciel au téléphone, l'interaction avec le client ne fait pas que se déplacer: elle se transforme. Dans le mouvement d'inscription (de l'oral à l'écrit), certains éléments sont retenus, fixés sous forme électronique, agencés dans des espaces précis. En changeant de support, ils deviennent des informations pertinentes qui ont pour vocation d'être mobilisées pour alimenter la prochaine conversation et opérer du coup un nouveau basculement de l'écrit à l'oral.

Ces basculements d'un support à l'autre opèrent une articulation des registres de l'activité. Ici, ils permettent de combiner dans la relation une temporalité longue (traces des interactions passées) et une temporalité courte (interaction directe rendue plus « efficace »). Dans d'autres cas, la trace de la relation-client peut être mise au service d'une interaction à plus long terme. Certaines informations sont ainsi enregistrées, puis réactivées pour élaborer des relances de clientèle personnalisées.

\begin{abstract}
Chaque client que l'on a n'est pas un client acquis, il y a énormément de travail, ce sont des personnes parfois que l'on suit depuis longtemps Pourquoi ? Parce qu'ils n'ont pas de besoin actuellement ou parce que ce client n'avait pas les connaissances de l'existence de notre entreprise sur Toulouse, et puis ils nous disent « re-contactez moi dans six mois... ». Un besoin pour développer un projet dans une société c'est pas comme un besoin «j'ai besoin d'un photocopieur... », on ne peut pas dès qu'on appelle, le besoin n'est pas tout le temps au moment... (...) Voilà, donc bon, maintenant les commerciales bossent un petit peu sur leur logiciel, assurent le suivi : rappeler au bout d'un certain temps, mais sans les " harceler» non plus! Si ils demandent de les rappeler dans six mois, hop, un petit message s'affiche au bout de quelques temps sur leur agenda, et sans attendre six mois on les rappelle... Sinon ils peuvent nous oublier aussi... (Assistante commerciale, SS2I)
\end{abstract}

Ces premières formes de chaînage mettent en lumière l'articulation de deux supports de communication (oral à distance et écrit électronique) et l'épaississement temporel de la relation-client qu'elle favorise. Mais cette articulation est loin de s'arrêter à l'aménagement de l'interaction directe avec le client. Certains chaînages participent à rendre possible et à aménager la circulation des informations qui concernent les clients au sein de l'entreprise.

\title{
La circulation du client et de la relation-client
}

\section{Recevoir les clients}

Lorsqu'un client prend contact avec une entreprise, la personne qui l'accueille n'est pas toujours celle qui est susceptible de répondre à sa demande. Dans ce cas, la chaîne technique peut s'enrichir d'outils qui permettent de faire circuler la demande jusqu'à l'interlocuteur adéquat. Lorsque cette personne est en mesure de répondre dans l'instant, un simple transfert de ligne téléphonique ou l'accompagnement du client dans le bon bureau suffisent. En revanche, lorsque l'interaction avec le client devient asynchrone, une série d'équipements variés fait son apparition. Au coup de téléphone et aux éventuelles inscriptions d'informations déjà évoquées vient par exemple s'ajouter l'utilisation en interne du téléphone portable et éventuellement de sa fonction " boîte vocale » lorsque la personne concernée est à l'extérieur de l'entreprise (c'est le cas de l'entreprise de plomberie, ou de l'agence immobilière).

Dans le lieu même de l'entreprise, d'autres systèmes peuvent être mis en place qui assurent le déplacement des informations en interne. C'est ce qui se passe dans l'entreprise de vente de logiciels médicaux où tout commence par le standard. Dans une situation de travail "normale " (c'est-à-dire quand le nombre d'appels n'est pas trop important), l'appel d'un client engage une réponse directe de la standardiste. Si elle peut régler le problème, s'il s'agit d'une question dont elle possède la réponse, la chaîne s'arrête là, le client est renseigné. Si ce n'est pas le cas, elle prend note du problème du 
client sur une fiche bleue qu'elle posera sur le bureau du technicien. Le technicien devra alors rappeler le client pour régler le problème, puis tenir la standardiste au courant.

On voit bien ici comment les chaînes techniques peuvent faciliter l'entrée et la circulation du client dans l'entreprise. Toutefois, au-delà même des nuances qu'il faut apporter aux discours du management qui invoquent un client toujours plus présent et plus puissant dans les organisations ${ }^{10}$, l'étude de ces chaînages montre que ce mouvement n'est pas à sens unique. Si certaines combinaisons d'outils de communication peuvent effectivement participer à l'intégration de la relation-client par-delà l'interaction avec un standard téléphonique ou une borne d'accueil, d'autres se présentent au contraire comme une opportunité pour construire une frontière plus nette entre les clients et l'entreprise.

\section{Tenir les clients à l'écart}

Jusqu'ici, nous avons vu comment l'équipement de la relation-client permettait essentiellement d'enrichir les interactions, que ce soit en accentuant la réactivité de l'entreprise, en activant pendant l'échange une série d'informations supplémentaires, ou en facilitant la circulation du client et de ses demandes dans l'entreprise. Les situations de surcharge dans l'entreprise qui vend des logiciels médicaux nous invitent toutefois à regarder dans la direction opposée. Les désignations du client qui sont inscrites dans les chaînes de technologies de communication y apparaissent en effet comme un moyen de vider l'interaction de sa chair et de refuser l'entrée de certains clients.

Dans cette entreprise, la chaîne évoquée plus haut peut prendre une tout autre forme, dès lors que le nombre d'appels reçus est jugé trop important. Un système y a été mis en place, à partir de l'aménagement de CustomScreen, qui permet de désigner le client au moment même de son appel. L'utilisation principale qui en est faite vise à segmenter la clientèle en deux catégories : ceux qui sont sous contrat avec l'entreprise et les autres. Les jours d'affluence, cette distinction est opérationnalisée et le dispositif permet " de prioriser » (nous dit la standardiste) les appels. Seule la fiche des clients qui sont sous contrat apparaît à l'écran. La standardiste se charge alors de répondre à leurs demandes et la chaîne décrite plus haut démarre. En revanche, les clients qui ne sont pas sous contrats sont automatiquement basculés vers un répondeur sur lequel ils sont invités à laisser leurs coordonnées. Celles-ci sont récupérées plus tard par la standardiste qui les fait suivre à la commerciale par voie orale ou par voie écrite, sur une petite fiche. Cette dernière aura alors en charge de démarcher le client ultérieurement.

Ici, la mobilisation automatique de la caractérisation des clients, qui engage un découpage radical de la clientèle, s'effectue par une combinaison de dispositifs (téléphone/logiciel de gestion des appels/répondeur) qui vient s'ajouter à une chaîne déjà longue. Cette articulation permet de mettre en place un filtrage des contacts de l'entreprise qui ne concerne pas d'ailleurs uniquement les clients. Cet usage nous renseigne sur la relation-client qui est supportée dans ce cas par les TIC. II n'est plus question ici de mettre les outils de communication au seul service d'une interaction " améliorée » avec le client. C'est essentiellement au nom de l'entreprise (et non plus de la relation) que le découpage et son opérationnalisation sont faits.

C'est la seule distinction qu'on va faire chez le client. Tout simplement parce que nous c'est notre façon de se rémunérer. Et si on répond à des personnes qui ne sont pas sous contrat on y perd. (Standardiste, entreprise de logiciels médicaux)

L'équipement du travail relationnel par la normalisation et l'automatisation de la caractérisation de la clientèle peut donc être aussi un moyen de lutter contre les débordements. On touche ici peut-être à une des spécificités des petites entreprises. Le

\footnotetext{
${ }^{10}$. Sur ce point, voir notamment J.-P. Neuville (1999), ou encore L. Caby et C. Jaeger (1998) pour la place que peuvent tenir les NTIC dans ce mouvement.
} 
faible nombre de salariés pèse en effet un poids non négligeable dans les possibles débordements auxquels l'entreprise doit faire face. Une seule standardiste ou secrétaire, voire une personne qui cumule différentes activités dont celle d'accueil, se trouvent vite submergées par les coups de téléphone ou les visites sur place dès lors que le nombre de clients potentiels atteint un certain seuil. Si dans le second cas, il n'est pas possible de caractériser le client sans entrer en contact avec lui, dans le premier certains outils le permettent. Leur utilisation mobilise cette caractérisation faite en amont pour canaliser la clientèle, et en rediriger une partie à l'extérieur de l'entreprise ou à sa périphérie technique (le répondeur). Bien entendu cette forme de mise à distance ne remet pas en question les usages mis en évidence dans le point précédent. Elle vient en revanche les compléter : mieux connaître et reconnaître le client permet parfois de mieux l'éviter.

L'équipement de la relation-client par les chaînages techniques est donc à double tranchant. L'accueil personnalisé, une meilleure intégration du client dans l'entreprise ne sont pas des valeurs " intrinsèques " aux $\mathrm{TIC}^{11}$. Certains usages montrent à quel point ils peuvent être mis au service de relations minimisées voire éludées. Selon les situations, les chaînes techniques qui permettent d'élaborer en amont une présentation de l'interlocuteur apparaissent tantôt comme des supports de l'implication des entreprises dans l'interaction avec le client, tantôt comme des supports de leur " désimplication ». La relation interpersonnelle "fusionnelle " avec le client n'est donc pas l'unique forme de lien qui a cours dans les petites entreprises et les chaînes techniques permettent de jouer sur les deux tableaux de la communication personnalisée et de l'interaction " froide ", pré-cadrée.

Les chaînages étudiés ici concernent enfin un dernier registre. À la rapidité de réaction, la trace de la relation, et la circulation des informations sur le client s'ajoute la mise en place de sauvegardes à plus long terme.

\section{Les figures de la sauvegarde}

La sauvegarde est une dimension importante des opérations communicationnelles des TPE que nous avons étudiées. On peut en dessiner trois figures. Elle participe tout d'abord à la traçabilité des activités de l'entreprise et de son stockage. De ce point de vue, le travail de sauvegarde est très proche de celui évoqué plus haut à propos de la relation-client.

[Notre base de données d'appels] nous permet aussi de faire des statistiques. (...) On s'en sert pour savoir combien on a d'appels par jour, et bon ça nous permet de savoir si on a besoin de personnes supplémentaires ou pas. Et un peu de savoir quand est-ce que se concentrent les appels. (Standardiste, entreprise de vente de logiciels médicaux)

Dans ce cas, la chaîne téléphone/logiciel de base de donées/statistiques est un moyen de participer à l'organisation du travail. Bien sûr on est loin ici de l'industrialisation des données de l'activité et des dérives auxquelles elles peuvent mener ${ }^{12}$, mais le principe est le même. Les données produites par l'informatisation de certaines informations offrent des repères pour (re)cadrer les modalités de travail.

La sauvegarde permet aussi de mettre en place une mémoire de l'entreprise. Cette mémoire à plus ou moins long terme se focalise sur l'enregistrement des données dont dispose l'organisation à des fins de conservation. Elle prend souvent la forme d'une trace qui s'ajoute à celles qu'opèrent les outils usuels de l'entreprise.

11 Cet argument rejoint celui de J.-P. Neuville et C. Musselin (2001) qui soulignent que l'« encatrement " ou le " désencastrement " social des relations marchandes ne sont pas inscrits dans les TIC a priori.

12. Pour un exemple d'intensification du travail, dans le cadre des hypermarchés, via la mise en œuvre de données statistiques informatisées qui tracent l'activité, voir S. Prunier-Poulmaire (2000). 
On a un système où toutes nos données sont sauvegardées tous les midis. Par exemple, si on a enregistré, je ne sais pas, par exemple de nouvelles bases de données, de midi à deux heures tout s'enregistre sur des gros ordinateurs, comme ça si on a un problème ici... (Agent d'affrètement, entreprise de fret)

Cette trace « en plus » peut aussi se mettre en place par l'intermédiaire d'un changement de support. C'est le cas dans l'entreprise de plomberie où les fiches-clients sont inscrites sur l'ordinateur, mais aussi sur un cahier, pour pallier les éventuelles pannes informatiques.

Enfin, le troisième type de sauvegarde est fondé sur des contraintes juridiques. Une trace de l'activité ou de la relation avec les clients est nécessaire pour parer aux situations de litiges ou de mises en cause de l'entreprise. Cette trace mobilise la plupart du temps de nouveaux maillons dans la chaîne.

[Ça vous sert à quoi en fait de garder les dossiers physiques ?] C'est obligatoire. Un dossier on doit le garder... Si vous voulez, on a un cahier avec les affaires faites, et un numéro pour chaque affaire. Et après dans chaque dossier il y a le fax de confirmation, le fax du transporteur, le prix, la CMR... Et tout ça c'est conservé pendant des années. [Vous avez un cahier où vous répertoriez chaque affaire ?] Oui. Chacun marque son affaire, à chaque fois. De toute façon c'est chronologique. On marque dessus le numéro d'ordre, le client, le transporteur, le lieu de départ, le lieu d'arrivée, le prix, le prix vendu. [Et il vous sert à quoi ce cahier ?] À faire des recherches, aussi pour les factures. Et puis en cas de litige. On nous dit telle affaire, tel numéro, il y a eu un problème, et alors on recherche sur le cahier. On va rechercher le dossier, et puis on regarde. (Directeur, entreprise de fret)

Dans cette logique d'action, où il est question de rendre des comptes et surtout de faire preuve, l'écrit et le support papier tiennent une place centrale.

Si un type t'écrit, tu as la lettre, tu l'archives, tu l'as en dossier papier. II y en a qui scannent et qui la détruisent. Moi, je la garde, parce que si un jour je dois la ressortir, on ne me dira pas que c'est un document que j'ai inventé. (Gérant, agence immobilière)

Le papier apporte aux différentes chaînes mises en place des dimensions très spécifiques. II opère tout d'abord une certaine matérialisation des informations. En ce sens, il assure une forme de stockage différente de celle que propose le support électronique (souvent considéré comme «immatériel »). En combinant papier et dispositifs informatiques, les petites entreprises peuvent donc mobiliser dans une même opération cette forme de stock matériel et immuable et la fluidité des supports de circulation numérisés.

Mais le support papier offre un autre avantage dans le régime de la preuve: il est le véhicule d'un acte d'écriture bien particulier: la signature. C'est sur elle que repose l'authentification et l'identification des documents et des personnes ${ }^{13}$. L'authentification des actes et l'engagement des personnes sont actualisés par leur signature, et celle-ci n'a de valeur aux yeux des entreprises qu'associée au support papier.

Mettons, vous avez une affaire à faire, vous joignez la personne, c'est bon, vous joignez le transporteur, et une fois que c'est fait, vous envoyez un fax à chacun. Le client vous envoie un fax pour vous dire l'affaire va se faire tel jour, dans telles conditions, et vous renvoyez une copie du fax au transporteur, sans le nom du client bien sûr, pour confirmation. (...) Le gars reçoit son fax, et il le renvoie avec un coup de tampon " bon pour accord ». Et vous gardez ça dans le fameux dossier physique, vous mettez ça dans le dossier de transport. (...) Dessus, il y a un numéro, le transporteur remplit sa partie, le client remplit sa partie. C'est signé au départ, c'est signé à l'arrivée, et s'il n'y a pas de litige, il n'y a rien, c'est

${ }^{13}$. FRAENKEL, 1992 
rajouté au dossier. En fait c'est un document officiel du transport. (Directeur, entreprise de fret)

Ces deux dimensions que les personnes attribuent au papier (matérialisation et signature) lui donne une place à part dans les chaînes techniques, à la manière du téléphone du côté de l'interaction avec les clients. Comme ce dernier, le support papier est en effet fortement ancré dans des usages où la confiance joue un rôle central. Dans le cas du téléphone, cette confiance tient dans la relation "directe », dialogique rendue possible par l'outil. La confiance qu'inspire le papier se situe à l'opposé. Elle repose sur sa pérennité et sur sa capacité à être mobilisé en tout temps et en tout lieu; et elle est encastrée dans des dispositifs juridiques qui définissent précisément les usages de ce support.

Les différentes chaînes techniques mises en œuvre dans les TPE permettent donc d'articuler des registres d'activité différents dans la relation avec les clients : rapidité dans l'accueil du client, réactivité, mémoire des échanges, circulation des informations dans l'entreprise, sauvegardes internes, traces juridiques... Ces articulations montrent à quel point la relation client/entreprise est plurielle. Elles montrent aussi que cette pluralité doit beaucoup à la matérialité que chaque support de communication attribue aux échanges et aux informations. C'est l'hétérogénéité de ces supports qui fait la force des chaînes techniques. Le téléphone est par exemple le véhicule d'interactions dialogiques orales, dans lesquelles un «ton" peut passer, la réaction de l'interlocuteur peut être appréhendée instantanément, les ajustements se faire par tâtonnements rapides, etc. ; les petits papiers manuscrits facilitent une circulation rapide d'informations éphémères pour des activités à brève échéance; l'inscription électronique de caractéristiques standardisées permet certaines manipulations de l'information comme le tri automatique dans un tableur... Chacun des supports fait ainsi jouer des ressources et des contraintes communicationnelles fortes qui composent un cadre technique pour l'échange et la circulation des informations. Le chaînage des dispositifs permet d'entrelacer ces cadres, et de profiter par exemple dans une même relation des possibilités offertes par les échanges électroniques, tout en passant par certaines étapes où circulent des documents sur papier ${ }^{14}$.

Les chaînes techniques qui s'élaborent à l'interface du client et de l'entreprise équipent donc une relation-client pluridimensionnelle qui déborde largement de l'interaction immédiate. Parce qu'elles combinent différents supports et donc différentes mises en forme de l'information, elles concilient dans une même relation des dimensions souvent antagonistes qui, sans elles, seraient source de tensions et nécessiteraient une hiérarchisation plus ou moins systématique.

\footnotetext{
${ }^{14}$. Ce point soulève la question de la position que l'informatique semble vouloir prendre aujourd'hui dans les entreprises, grandes ou petites, via les nouveaux dispositifs de communication et de gestion. Les systèmes de CRM ou d'ERP tendent en effet vers des modèles de d'organisation de l'information centrés sur des dispositifs « tout en un » capables de cristalliser toutes les opérations de la relation client pour les premiers, toute la gestion de l'entreprise pour les seconds. Du point de vue d'une problématique des chaînes techniques, ce type d'outils se positionne comme se substituant (en la rationalisant) à la totalité de la chaîne. L'hétérogénéité matérielle n'y est pas considérée comme opportune, quand elle n'est pas directement stigmatisée comme un éparpillement, une perte de temps et d'argent. II est fort à parier que la mise en place de tels systèmes intégrateurs reproduise des situations bien connues en ergonomie, où les personnes, confrontées ici à un dispositif unique de communication officielle, élaborent des activités parallèles et invisibles qui permettent de reconstituer des chaînes malgré tout.
} 


\section{Conclusion : l'épaisseur de la relation}

La perspective adoptée ici s'est focalisée sur la question des usages et celle des valeurs qui sont attachées aux dispositifs de communication ${ }^{15}$. Elle a permis de montrer que les chaînes techniques mobilisées dans l'activité quotidienne des petites entreprises participaient à articuler des registres différents au fil d'une même relation. Pour qualifier ces registres, j'ai essayé de saisir le type de temporalité qui était en jeu dans l'usage de chaque dispositif au sein de la chaîne. Bien entendu - et c'est apparu au fur et à mesure des exemples et de leur analyse - ces registres relationnels débordent largement le seul cadre temporel. Ils sont autant de manières de faire lien dans les échanges entre les entreprises et leurs clients.

Comme le soulignait l'introduction de cet article, dans les petites entreprises, les relations qui "sautent aux yeux" de l'observateur étranger sont celles qui se fondent sur des échanges interpersonnels, des rencontres informelles qui débordent de la rencontre marchande telle qu'elle est modélisée par les économistes classiques. On y connaît des « bons clients » depuis longtemps, et les conversations que l'on a avec eux ne s'arrêtent pas à l'échange d'informations sur les produits ou sur les prix. Ce point illustre directement l'argument, désormais classique, de l'encastrement social des marchés. Les échanges marchands sont ancrés dans des réseaux de relations sociales qui les dépassent et les nourrissent. Ces relations sont la base de liens durables, sur lesquels repose la confiance des deux parties et qui réduisent en partie les coûts qui seraient nécessaires à la recherche de nouveaux partenaires.

Mais une analyse microsociologique attentive aux dispositifs communicationnels, telle qu'elle a été développée ici, montre que cette première vision ne suffit pas. Tout d'abord, la tentation paraît grande de faire de ces relations "informelles " des interactions sociales "pures", sur le modèle de la socialisation primaire de Simmel ${ }^{16}$. Outre le fait que cette " pureté » semble impossible à saisir à moins d'oublier le cadre situationnel de toute rencontre, l'étude des chaînes techniques montre que le téléphone, le fax, ou encore le courrier électronique, prennent une part considérable dans la possibilité de maintenir ce type de lien ${ }^{17}$. En d'autres termes, même les relations sociales les plus " chaudes ", les plus personnelles, sont équipées. Ce point apparaît notamment dans les grandes entreprises où l'on cherche précisément à reconstruire ce type de relations chaleureuses via des dispositifs communicationnels et organisationnels complexes ${ }^{18}$.

Mais la tentation est plus grande encore semble-t-il de considérer que cette forme de lien " privilégié » soit la seule qui ait cours dans les petites entreprises. La force de l'argument de l'encastrement social des marchés a tendance à laisser dans l'ombre d'autres types de liens, moins personnels, pourtant omniprésents dans les activités marchandes quotidiennes $^{19}$. Nous avons vu que, précisément parce qu'ils sont pris dans des chaînes techniques communes, ces liens formels et impersonnels (comme l'échange de documents signés ou la figuration sous forme de codes dans un logiciel de gestion) sont non seulement fréquents dans les petites entreprises, mais sont totalement indissociables

\footnotetext{
15. Cette perspective est fortement guidée par la méthode elle-même qui consiste a interroger les personnes sur l'utilisation qu'elles font de différents dispositifs communicationnels. Une telle entrée en matière donne un cadre à l'entretien qui favorise la production d'un discours centré sur ces dispositifs et leurs valeurs pour l'action. Ce type d'enquête gagnerait à s'étoffer, en devenant combinatoire, pour compléter les dimensions exposées ici d'une analyse des situations quotidiennes de communication.

16. SIMMEL, 1981.

17. Une enquête en cours montre que les logiciels de messagerie instantanée semblent prendre une place nouvelle et très importante dans ce processus.

18. MALLARD, 2002.

19. CHANTELAT, 2002.
} 
de leurs pendants informels ${ }^{20}$. Le chaînage des dispositifs et l'hétérogénéité des supports qu'il permet de combiner dessinent un continuum entre formel et informel, échanges " froids » et " chauds », au sein d'une même relation. Les registres relationnels ne sont pas à sens unique. La combinaison quotidienne des dispositifs communicationnels opérationnalise leur diversité.

20. L'enquête présentée ici s'est limitée à des situations statiques où les usages sont pour la plupart stabilisés. Elle s'ouvre sur l'intérêt qu'il y aurait à suivre des chaînes techniques dans la durée pour comprendre comment l'environnement sociotechnique auquel elles participent est susceptible d'évoluer, et saisir les éventuelles tensions qui peuvent y voir le jour, notamment au regard des innovations technologiques qui ne cessent de l'alimenter et des « modèles relationnels » qu'elles tentent d'y imposer. 


\section{Références}

CABY L. \& JAEGER C. (1998), « La relation fournisseur-client et les technologies de l'information et de la communication », Réseaux, 91, p. 96-117.

CHANTELAT P. (2002), " La Nouvelle Sociologie Économique et le lien marchand : des relations personnelles à l'impersonnalité des relations », Revue française de sociologie, 43-3, p. 521-556.

FRAENKEL B. (1992), La signature : genèse d'un signe, Paris, Gallimard.

FRAENKEL B. (2001), "Enquêter sur les écrits dans l'organisation », in A. Borzeix et B. Fraenkel (dir.) Langage et travail : communication, cognition, action, Paris, CNRS éditions, p. 231-261.

GRANOVETTER M. (1985), « Economic action and social structure: the problem of embeddedness », American journal of sociology, 91-3, p. 481-510.

GROSJEAN M. \& LACOSTE M. (1998), « L'oral et le tout écrit à l'hôpital », Sociologie du travail, XL-4, p. 439-465.

LÉVY E. (2002), " L'usager est-il soluble dans l'organisation ? », Sciences de la Société, 56, p. $186-202$.

LICOPPE C. (2001), « Faire ses courses par téléphone ou par internet. Médiations techniques, formes des échanges, de la relation commerciale et de la consommation », Réseaux, 106, p. 73-101.

MALLARD A. (2002), " Les nouvelles technologies dans le travail relationnel : Vers un traitement plus personnalisé de la figure du client ? », Sciences de la Société, 56, p. 6277.

NEUVILLE J.-P. (1999), « Le marché et la convention à l'épreuve de l'organisation », Sciences de la société, 46, p. 103-117.

NEUVILLE J.-P. \& MUSSELIN C. (2001), « Introduction au numéro spécial NTIC », Sociologie du travail, 43-3, p. 303-308.

NORMAN D. (1993), " Les artefacts cognitifs », in B. Conein, N. Dodier et L. Thévenot (dir.) Les objets dans l'action. De la maison au laboratoire, Paris, Éditions de l'EHESS. Coll. Raisons pratiques $n^{\circ} 4$, p. 15-34.

PRUNIER-POULMAIRE S. (2000), «Flexibilité assistée par ordinateur. Les caissières d'hypermarché », Actes de la recherche en sciences sociales, 134, p. 29-36.

SIMMEL G. (1981), Sociologie et épistémologie, Paris, PUF. 